\title{
A UTILIZAÇÃO DOS SOFTWARES GEOGEBRA, WINPLOT E K3DSURF NO ESTUDO DAS SUPERFÍCIES QUÁDRICAS
}

\section{THE USE OF SOFTWARE GEOGEBRA, WINPLOT AND K3DSURF IN THE SURFACE QUADRIC}

\author{
Danielle Apolinário da Silva ${ }^{1}$ \\ Universidade Federal da Paraíba - UFPB \\ Claudilene Gomes da Costa ${ }^{2}$ \\ Universidade Federal da Paraíba - UFPB
}

\begin{abstract}
Resumo
O presente trabalho teve como objetivo geral apresentar um estudo capaz de contribuir e facilitar a aprendizagem de conceitos de Geometria Analítica e o desenvolvimento da visualização geométrica de objetos tridimensionais, a partir da utilização dos softwares educacionais Geogebra, Winplot e K3DSurf. O estudo foi desenvolvido com os alunos da disciplina de Cálculo Diferencial e Integral III, do curso de Licenciatura em Matemática, da Universidade Federal da Paraíba/Campus IV, em Rio Tinto-PB, surgindo como elemento de apoio diante das dificuldades de aprendizagem que esses alunos apresentam no que diz respeito ao estudo das quádricas. A metodologia utilizada para o desenvolvimento da pesquisa caracteriza-se em relação aos objetivos como exploratória e descritiva. Quanto aos procedimentos técnicos utilizados caracteriza-se como pesquisa bibliográfica e estudo de caso, e em relação ao método de abordagem, caracteriza-se como uma pesquisa qualitativa e quantitativa. Já os instrumentos empregados na coleta de dados da pesquisa foram atividades realizadas com o auxílio dos softwares e um questionário diagnóstico, contendo vinte questões fechadas. Com relação aos resultados, observou-se que os alunos mesmo estando em um curso de Licenciatura em Matemática apresentam algumas dificuldades de aprendizagem de conceitos de Geometria Analítica, especificamente no que se refere à visualização e construção gráfica de superfícies. Entretanto, os dados obtidos através da aplicação das atividades permitem afirmar que os softwares Geogebra, Winplot e K3DSurf se caracterizam como um instrumento que contribui de modo significativo para que essas dificuldades sejam elucidadas. Verificou-se ainda que esses softwares facilitam, principalmente, a visualização gráfica das superfícies, além de permitir o estudo dos elementos e propriedades das quádricas, a partir de suas potenciais ferramentas de construção e animação. Ainda dentre os resultados, os alunos puderam opinar sobre o uso dos softwares para o estudo de conteúdos matemáticos em outras disciplinas do curso. Todos afirmaram que o suporte dos softwares Geogebra, Winplot e K3DSurf, poderiam também contribuir e facilitar a aprendizagem em outras disciplinas do Curso de Licenciatura em Matemática.
\end{abstract}

\footnotetext{
${ }^{1}$ danyapolinario@ hotmail.com

2 claudilene@dce.ufpb.br
} 
Palavras-chave: Software Geogebra; Software Winplot; Software K3dsurf; Quádricas; Ensino da Matemática.

\begin{abstract}
The present work had as general objective to present a study capable of contributing to and facilitating a learning of concepts of Analytical Geometry and geometric development of three - dimensional objects, using the computational software Geogebra, Winplot and K3DSurf. The study was developed with the students of the discipline of Differential and Integral Calculus III, a course of Mathematics Degree, of the Federal University of Paraíba / Campus IV, in Rio Tinto-PB, appearing as an element of support to the learning difficulties that students presents regarding the study of quadrics. The methodology used for the development of the research is characterized in relation to objectives as exploratory and descriptive. As for the technical procedures used are characterized as a bibliographic research and a case study and in relation to the method of approach, it is characterized as a qualitative and quantitative research. The instruments used in the data collection of the research were performed with the help of softwares and a diagnostic questionnaire, containing twenty closed questions. Regarding the results, it was observed that students even being in an undergraduate Mathematics degree course present some difficulties learning concepts of Analytic Geometry, specifically regarding to the visualization and graphical construction of surfaces. However, the data obtained on the application of the activities related to the software Geogebra, Winplot and K3DSurf are characterized as an instrument that contributes in a significant way so that the difficulties are elucidated. It was also verified that these softwares facilitate, mainly, a graphical perspective of the surfaces, besides of allowing the study of the elements and properties of the quadrics, from its potential tools of construction and animation. Still among the results, the students were able to comment on the use of the softwares to study mathematical contents in other disciplines of the course. All software related to Geogebra, Winplot and K3DSurf, also help and facilitate learning in other disciplines of the undergraduate Mathematics course.
\end{abstract}

Keywords: Geogebra Software; Winplot Software; K3dsurf Software; Quadric; Mathematics Teaching.

\title{
Introdução
}

No atual contexto social, de permanente processo de informatização, as tecnologias estão diretamente relacionadas à formação de ideias e comportamentos dos indivíduos. Segundo os Parâmetros Curriculares Nacionais - PCN "as tecnologias, em suas diferentes formas e usos, constituem um dos principais agentes de transformação da sociedade, pelas modificações que exercem nos meios de produção e por suas consequências no cotidiano das pessoas.” (BRASIL, 1998, p. 43). Dessa forma, vem surgindo nas instituições de ensino a grande necessidade de aperfeiçoamento nos 
métodos didáticos para o ensino e aprendizagem, tornando-se cada vez mais necessária a utilização de recursos tecnológicos.

A utilização de softwares educacionais para o ensino da Matemática pode desenvolver no aluno a capacidade de representação, interpretação, visualização, comprovação e demonstração de conceitos matemáticos. Segundo Ghiggi, Schmidt e Koch (2013, p. 04), “a incorporação de softwares educacionais como ferramenta de apoio ao ensino de matemática favorece a compreensão de conceitos, a exploração e relação de diversas representações, a exploração de propriedades e relações matemáticas". Dessa forma, os softwares aliados ao ensino de Matemática podem oferecer ao discente um meio eficaz para promover a aprendizagem.

Os softwares educacionais vêm adquirindo um espaço relevante na sala de aula, embora muitos docentes ainda não se sentem seguros para trabalhar com esse recurso, seja pela ausência de disciplinas direcionadas a utilização de tecnologias como recurso didático durante a sua formação e não procurarem fazer uma formação continuada, ou mesmo pelo o seu próprio comodismo.

No curso de Licenciatura em Matemática, especificamente nas disciplinas de Cálculo Vetorial e Cálculo Diferencial e Integral (CDI), a aprendizagem de conteúdos de Geometria Analítica vem enfrentando algumas dificuldades, decorrentes das necessidades apresentadas pelos discentes devido à ausência dos conteúdos de Geometria, especificamente da Geometria Analítica, durante o ensino básico, ou até mesmo pela própria desmotivação do aluno diante da metodologia adotada pelo professor em sala de aula.

Nessa perspectiva, o presente trabalho apresenta os resultados de um Trabalho de Conclusão de Curso que teve como objetivo geral apresentar um estudo capaz de contribuir e facilitar a aprendizagem de conceitos de Geometria Analítica e o desenvolvimento da visualização geométrica de objetos tridimensionais, a partir da utilização dos softwares educacionais Geogebra, Winplot e K3DSurf. A pesquisa foi desenvolvida com os alunos da disciplina de Cálculo Diferencial e Integral III, do curso de Licenciatura em Matemática, da Universidade Federal da Paraíba/Campus IV, em Rio Tinto-PB, surgindo como elemento de apoio diante das dificuldades de aprendizagem que esses alunos apresentam no que diz respeito ao estudo das superfícies quádricas. 


\title{
Tecnologias de Informação e Comunicação e o Ensino de Matemática
}

As tecnologias de informação e comunicação (TIC) vêm passando por um constante processo de modernização, e estão cada vez mais presentes nos ambientes educacionais, dentre as quais, os recursos de informática vêm adquirindo grande espaço e significação, principalmente pelo o fato da Internet ter se expandido para as escolas e universidades. De acordo com Valente e Almeida (1997, p. 01), "a História da Informática na Educação no Brasil data de mais de 20 anos. Nasceu no início dos anos 70 a partir de algumas experiências na UFRJ, UFRGS e UNICAMP”.

Valente e Almeida falam em seu trabalho sobre a influência de outros países no desenvolvimento da informática na educação brasileira:

\begin{abstract}
A Informática na Educação no Brasil nasce a partir do interesse de educadores de algumas universidades brasileiras motivados pelo que já vinha acontecendo em outros países como nos Estados Unidos da América e na França. Embora o contexto mundial de uso do computador na educação sempre foi uma referência para as decisões que foram tomadas aqui no Brasil, a nossa caminhada é muito particular e difere daquilo que se faz em outros países. Apesar das nossas inúmeras diferenças, os avanços pedagógicos conseguidos através da informática são quase os mesmos que em outros países. Nesse sentido estamos no mesmo barco. (VALENTE E ALMEIDA, 1997, p. 02).
\end{abstract}

Para Valentim (2014, p. 17), as TIC são responsáveis por mudanças profundas e positivas na educação, softwares, televisão, computadores, programas e etc., tornaram as aulas mais atrativas e mais dinamizadas [...].

Existem muitos recursos tecnológicos que oferecem ambientes de ensino nos quais a aprendizagem é transmitida de forma repetitiva, e o conhecimento é apenas memorizado e reproduzido. Mas, existem ferramentas que oferecem ao ensino e aprendizagem uma abordagem construtivista, e por meio delas o aluno passa a ser um sujeito ativo, podendo construir o próprio conhecimento matemático. "Nestes ambientes os alunos expressam, confrontam e refinam suas idéias, e 'programam' o computador sem precisar usar recursos de linguagem de programação [...]". (GRAVINA e SANTAROSA, 1998, p. 02).

Alguns recursos computacionais se constituem como importantes metodologias para o ensino e aprendizagem da Matemática, e podem conceder o desenvolvimento do pensamento matemático do aluno e a construção do seu conhecimento. 
De acordo com Gravina e Santarosa (1998, p. 08) "Os ambientes informatizados apresentam-se como ferramentas de grande potencial frente aos obstáculos inerentes ao processo de aprendizagem". Vários estudos constatam que o uso bem planejado de programas e aplicativos com fins educacionais contribui efetivamente para a aprendizagem, principalmente por envolver um fator que estar presente cada vez mais no dia a dia dos indivíduos: a tecnologia.

$\mathrm{Na}$ visão de Hebenstreint, o computador permite criar um novo tipo de objeto os objetos 'concreto-abstratos'. Concretos porque existem na tela do computador e podem ser manipulados; abstratos por se tratarem de realizações feitas a partir de construções mentais. (HEBENSTREINT 1987, apud GRAVINA e SANTAROSA, 1998, p. 08).

Dessa forma, a inserção de softwares educacionais no ensino e aprendizagem da Matemática pode desencadear o interesse pelo conteúdo matemático a ser estudado, tendo em vista que essas tecnologias podem favorecer ao aluno o estudo da matemática concreta e abstrata, e estão cada vez mais acessíveis e presentes em seu cotidiano. Com isso, havendo a possibilidade de um avanço significativo no processo de aprendizagem matemática.

Nessa perspectiva, os softwares Geogebra, Winplot e K3DSurf são programas que proporcionam ao usuário (aluno ou professor) os comandos para a construção do modelo matemático, levando-o a descobrir o significado real da Geometria Analítica, podendo favorecer o desenvolvimento da visualização geométrica através de seus recursos de controle e animação.

\section{Metodologia}

O presente trabalho teve sua metodologia desenvolvida para a aplicação e verificação por meio de uma pesquisa aplicada, que segundo Prodanov e Freitas (2013, p. 51), "objetiva gerar conhecimentos para aplicação prática dirigidos à solução de problemas específicos." Assim, a maior finalidade desta pesquisa é apresentar um estudo que venha a contribuir com o ensino e aprendizagem em relação ao conteúdo de superfícies quádricas e que traga soluções para as necessidades dessa aprendizagem.

Em relação aos objetivos a pesquisa caracteriza-se como exploratória e descritiva. É exploratória por visar uma familiarização com o problema a ser estudado, 
no caso dessa pesquisa, o estudo das quádricas, com a finalidade de apresentar um estudo que venha a contribuir e facilitar a aprendizagem de conceitos de Geometria Analítica e o desenvolvimento da visualização geométrica de objetos tridimensionais, a partir da utilização dos softwares educacionais Geogebra, Winplot e K3DSurf. E descritiva por pretender descrever se o uso de softwares no ensino das quádricas é um recurso facilitador para a aprendizagem e visualização das quádricas.

Quanto aos procedimentos técnicos utilizados, a pesquisa classifica-se como pesquisa bibliográfica e estudo de caso. A pesquisa bibliográfica foi utilizada para nortear a realização da fundamentação teórica, tendo alguns meios para consultas sobre o que já foi estudado e publicado a respeito do estudo das quádricas. Já o estudo de caso foi utilizado por buscar apresentar um estudo aprofundado sobre as quádricas a partir da utilização de softwares educacionais. A esse respeito, Gil enfatiza que o estudo de caso "é caracterizado pelo estudo profundo e exaustivo de um ou de poucos objetos, de maneira a permitir o seu conhecimento amplo e detalhado [...]”. (GIL, 2008, p. 57).

Em relação ao método de abordagem, a pesquisa caracteriza-se como qualitativa e quantitativa. Na abordagem qualitativa, a pesquisa tem o ambiente como fonte direta dos dados. O método quantitativo foi utilizado na pesquisa com a finalidade de facilitar a compreensão e interpretação dos dados.

Assim, o estudo foi realizado na Universidade Federal da Paraíba/Campus IV, em Rio Tinto-PB, visto que é um espaço que apresenta pessoas que possuíam as características fundamentais para o desenvolvimento da nossa pesquisa, dessa forma, nos fornecendo a amostra necessária.

Foram tomados como amostra da pesquisa 20 alunos do terceiro período do curso de Licenciatura em Matemática, da disciplina de Cálculo Diferencial e Integral III, do horário noturno. Com isso, a pesquisa foi desenvolvida a partir da coleta de dados favorecida por meio da aplicação de um questionário diagnóstico acerca do estudo proposto na oficina pedagógica aplicada, com a finalidade de verificar, principalmente, o desempenho dos alunos com o conteúdo de superfícies quádricas a partir da utilização dos softwares educacionais Geogebra, Winplot e K3DSurf, e se o uso desses softwares pode ser um recurso facilitador para o estudo das quádricas.

\section{Análise e discussões dos resultados}


A oficina pedagógica foi aplicada com os alunos da disciplina de Cálculo Diferencial e Integral III, do período 2016.2, do curso de Licenciatura em Matemática. Sendo realizada no LEPEM - Laboratório de Estudos e Pesquisas em Ensino de Matemática da Universidade Federal da Paraíba, Campus IV. A oficina teve duração de 180 minutos.

$\mathrm{Na}$ primeira atividade proposta, foi solicitada a identificação da superfície quádrica de equação $\frac{\mathrm{x}^{2}}{4}+\frac{\mathrm{y}^{2}}{5}+\frac{\mathrm{z}^{2}}{2}=16$ e a sua representação gráfica utilizando os três softwares. Na sequência, solicitava-se para representar nos softwares a quádrica de equação $\frac{x^{2}}{4}+\frac{y^{2}}{5}+\frac{z^{2}}{15}=16$, utilizando os mesmos coeficientes para as variáveis x e y da equação anterior, mudando apenas o coeficiente para a variável z, e a partir das duas representações foi questionado sobre o que ocorreu com a superfície.

A seguir, pode-se observar na figura 1 a representação gráfica obtida do primeiro elipsóide, pelo aluno A, no software Geogebra.

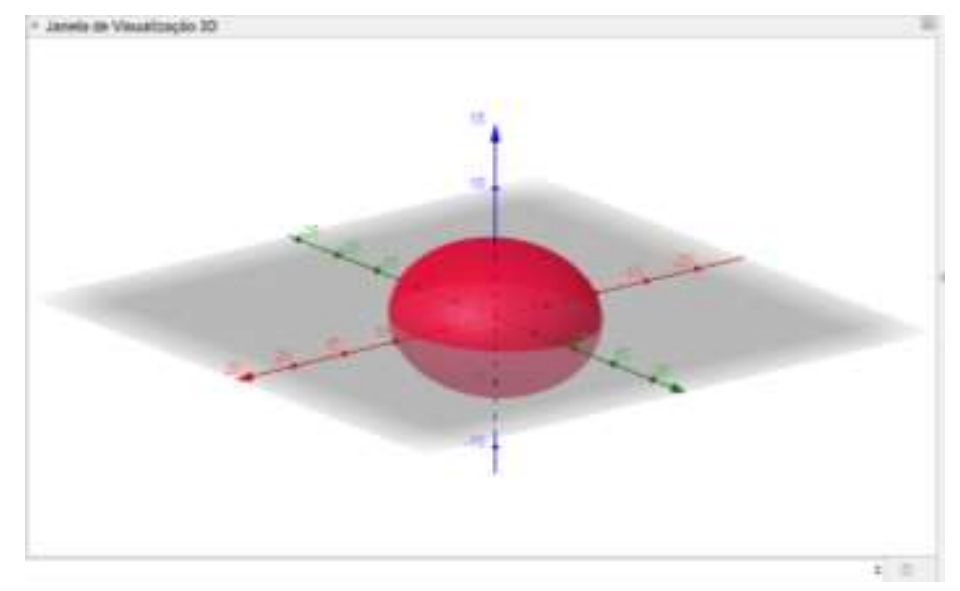

Figura 1 - Representação gráfica do elipsóide de equação $\frac{\mathrm{x}^{2}}{4}+\frac{\mathrm{y}^{2}}{5}+\frac{\mathrm{z}^{2}}{2}=16$

Dos alunos envolvidos no estudo, todos conseguiram identificar os elipsóides e representá-los nos softwares, visualizar e expressar o que ocorreu com as superfícies, ao ser modificada a variação para o eixo $\mathrm{Oz}$, além de comprovarem que a superfície quádrica é simétrica em relação a todos os eixos e planos coordenados e a origem.

A segunda atividade solicitava a construção do hiperbolóide de uma folha de equação $4 \mathrm{x}^{2}-4 \mathrm{y}^{2}+\mathrm{z}^{2}=4$, e do hiperbolóide de duas folhas dado pela equação $-4 \mathrm{x}^{2}-4 \mathrm{y}^{2}+\mathrm{z}^{2}=4$, nos softwares, utilizando-se os mesmos coeficientes para as variáveis $\mathrm{x}, \mathrm{y}$ e $\mathrm{z}$, diferenciando-se apenas o sinal do coeficiente da variável $\mathrm{x}$. 
Dessa forma, os alunos teriam que observar a relação entre as equações estabelecidas e as superfícies geradas, quanto à classificação, como mostram as representações realizadas no software K3DSurf, pelo aluno B, nas figuras 2 e 3.

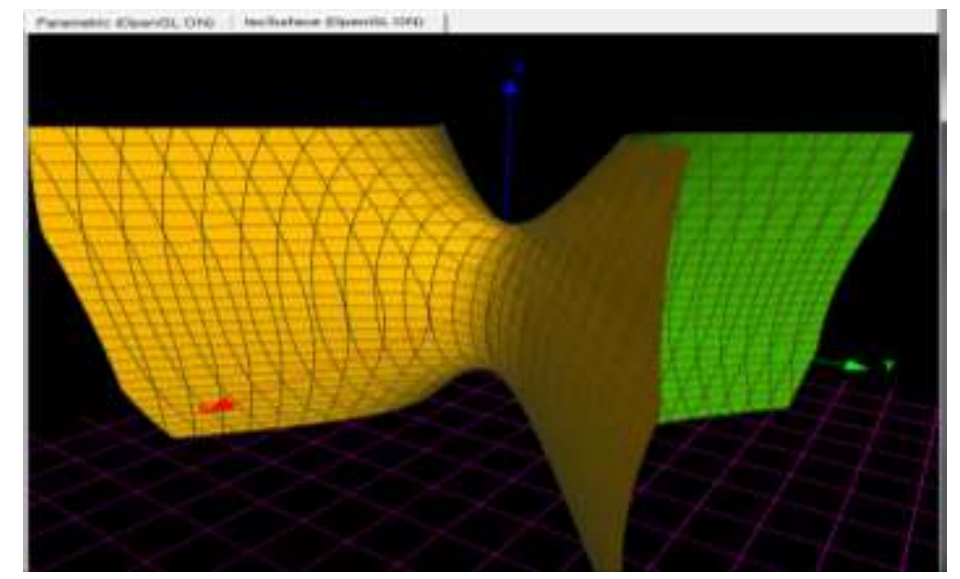

Figura 2 - Hiperbolóide de uma folha de equação $4 x^{2}-4 y^{2}+z^{2}=4$

Nessa primeira representação, todos os alunos observaram e afirmaram que quando a equação apresenta um coeficiente negativo para uma das variáveis, a superfície ficará em torno do eixo coordenado correspondente à variável.

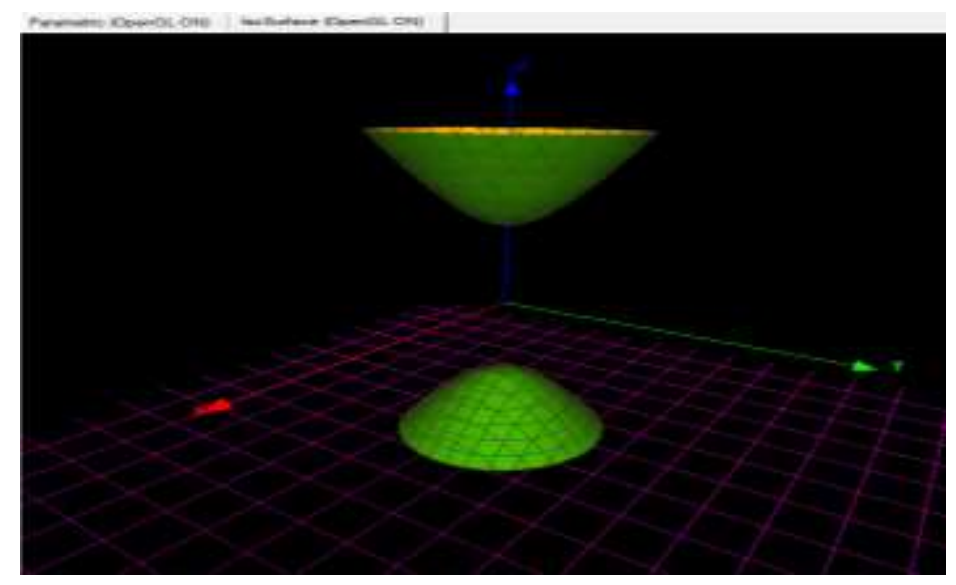

Figura 3 - Hiperbolóide de duas folhas de equação $-4 x^{2}-4 y^{2}+z^{2}=4$

Com essa atividade os alunos verificaram a relação entre as equações e os hiperbolóides gerados, quanto à classificação de cada um. Dessa forma, comprovaram as definições estudadas dos tipos de hiperbolóides, observando a relação entre os sinais dos coeficientes das variáveis e as superfícies obtidas. 
$\mathrm{Na}$ terceira atividade os alunos teriam que representar nos softwares três parabolóides e analisar a relação entre suas equações com os gráficos gerados, levando em consideração a classificação de cada um deles.

No primeiro item da terceira atividade, tem-se a equação de um parabolóide hiperbólico $\frac{x^{2}}{1}-\frac{y^{2}}{4}-z=0$, na qual o coeficiente da variável y apresenta sinal negativo e assim, a superfície é gerada tanto na parte inferior do plano como na superior e ao longo do eixo $\mathrm{Oy}$.

$\mathrm{Na}$ quarta atividade foi proposta a representação gráfica nos softwares, de hiperbolóides de uma folha, as quais apresentavam a mesma variação para $\mathrm{x}, \mathrm{y}$ e z e, diferindo-se apenas os sinais de cada uma. Feito isso, deveriam ser analisadas as características existentes entre os hiperbolóides.

A representação gráfica de um dos hiperbolóides de uma folha no software K3DSurf é apresentada na figura seguinte.

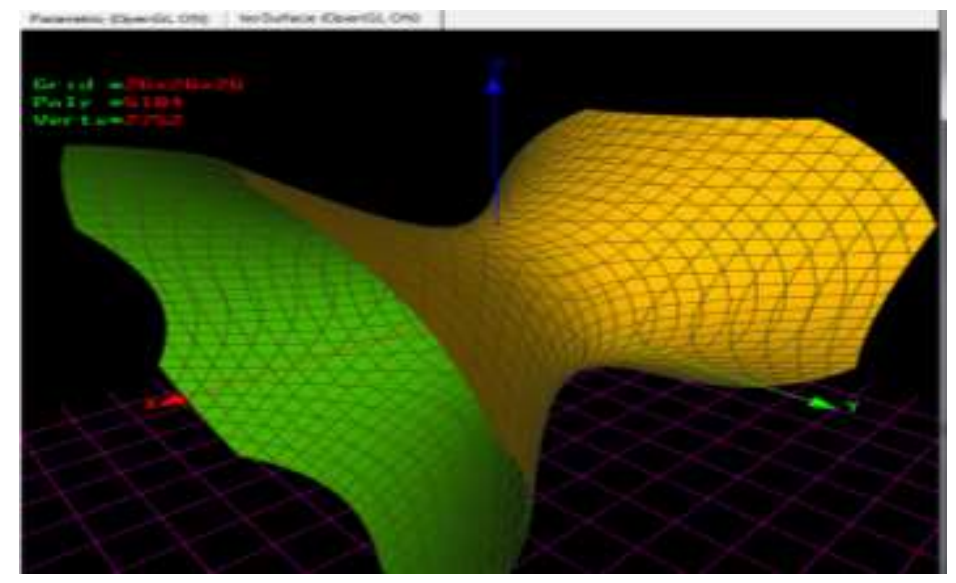

Figura 4 - Representação gráfica do hiperbolóide de uma folha de equação

$$
-\frac{x^{2}}{4}+\frac{y^{2}}{4}+\frac{z^{2}}{4}-1=0
$$

A quinta e última atividade, propôs aos alunos a identificação de algumas quádricas a partir de suas equações, e que encontrassem os seus traços e esboçasse-os em folhas ou no caderno. Em seguida, foram propostas suas construções nos softwares Geogebra, Winplot e K3DSurf.

Os alunos representaram graficamente as superfícies quádricas nos três softwares, mas obtiveram maior desempenho no Geogebra e no K3DSurf. Em alguns 
casos a superfície não foi gerada completamente no software Winplot, houve uma interrupção da continuidade entre a superfície, sobre o eixo Ox.

Dessa forma, verificou-se que os softwares educacionais Geogebra, Winplot e K3DSurf, proporcionaram aos alunos o estudo das características e propriedades das superfícies quádricas, por meio do qual investigaram e evidenciaram conceitos que já haviam estudado com o auxílio de recursos tradicionais de ensino, conceberam novos conhecimentos, além de terem aprimorado a visualização gráfica dos objetos tridimensionais estudados.

A análise dos dados obtidos através do questionário é apresentada por meio de tabelas, sendo elas referentes ao ambiente informatizado de ensino e à utilização de softwares educacionais; desempenho dos softwares Geogebra, Winplot e K3Dsurf no estudo das quádricas; metodologia que apresenta usabilidade, desenvolvimento da visualização e compreensão de conceitos; avaliação dos softwares para o estudo de quádricas; recurso facilitador que contribui para a aprendizagem e o estudo das quádricas a partir da utilização dos softwares. O percentual apresentado em todas as tabelas é referente aos 20 alunos envolvidos na pesquisa.

A tabela a seguir expõe questionamentos a respeito de qual software utilizado no estudo apresenta maior facilidade ou dificuldade e maior desempenho diante do estudo proposto.

Tabela 1: Utilização dos softwares Geogebra, Winplot e K3DSurf no estudo de quádricas

\begin{tabular}{cccccccc}
\hline Questionamentos & Geogebra & $\%$ & Winplot & $\%$ & K3DSurf & $\mathbf{\%}$ & $\begin{array}{c}\text { Total } \\
\%\end{array}$ \\
\hline $\begin{array}{c}\text { Qual (is) software } \\
\text { apresenta maior } \\
\text { facilidade para o estudo } \\
\text { das quádricas? }\end{array}$ & 17 & $85 \%$ & 2 & $10 \%$ & 1 & $5 \%$ & $100 \%$ \\
\hline $\begin{array}{c}\text { Qual (is) software } \\
\text { apresenta maior } \\
\text { dificuldade para o }\end{array}$ & 0 & $0 \%$ & 11 & $55 \%$ & 9 & $45 \%$ & $100 \%$ \\
estudo das quádricas? & & & & & & & \\
\hline $\begin{array}{c}\text { Qual (is) software } \\
\text { apresenta maior }\end{array}$ & 19 & $95 \%$ & 1 & $5 \%$ & 0 & $0 \%$ & $100 \%$ \\
desempenho diante do \\
estudo proposto?
\end{tabular}

Fonte: Estudo de Caso, 2017.

É possível observar na tabela que 17 alunos indicaram o Geogebra como o software que apresentou maior facilidade para o estudo das quádricas, indicando $85 \%$ 
do total de alunos envolvidos na pesquisa, $10 \%$ apontaram o Winplot e apenas 5\% alegaram que o K3DSurf apresenta maior facilidade para o estudo proposto.

Verifica-se ainda que o Winplot foi apontado como o software que apresenta maior dificuldade para o estudo das quádricas, representando um percentual de $55 \%$. Em segunda posição, encontra-se o K3Dsurf, representando $45 \%$ e por último o Geogebra, caracterizando $0 \%$ da amostra da pesquisa. A tabela ainda mostra que $95 \%$ dos alunos afirmaram que o software Geogebra apresenta maior desempenho diante do estudo proposto na oficina pedagógica, apenas 5\% indicam que é o software Winplot e $0 \%$ o K3Dsurf.

A tabela 2 expõe as percepções dos alunos sobre a utilização dos softwares Geogebra, Winplot e K3DSurf como recurso para a aprendizagem matemática.

Tabela 2: Os softwares Geogebra, Winplot e K3DSurf como recurso para a aprendizagem matemática

\begin{tabular}{ccccc}
\hline $\begin{array}{c}\text { Os softwares Geogebra, Winplot e } \\
\text { K3DSurf }\end{array}$ & Sim & Não & Total & \% \\
\hline $\begin{array}{c}\text { É um recurso facilitador para a } \\
\text { aprendizagem de quádricas? }\end{array}$ & 20 & 0 & 20 & $100 \%$ \\
\hline $\begin{array}{c}\text { Contribuem efetivamente para a } \\
\text { aprendizagem de quádricas? }\end{array}$ & 20 & 0 & 20 & $100 \%$ \\
\hline $\begin{array}{c}\text { Podem contribuir para a aprendizagem } \\
\text { de outros conteúdos matemáticos? }\end{array}$ & 20 & 0 & 20 & $100 \%$ \\
\hline $\begin{array}{c}\text { Oferecem benefícios se comparados } \\
\text { ao trabalho no papel e no quadro? }\end{array}$ & 20 & 0 & 20 & $100 \%$ \\
\hline $\begin{array}{c}\text { Podem contribuir e facilitar a } \\
\text { aprendizagem em algumas disciplinas } \\
\text { do curso? }\end{array}$ & 20 & 0 & 20 & $100 \%$ \\
\hline Fon Esolo Cas, 2017. & & & \\
\hline
\end{tabular}

Fonte: Estudo de Caso, 2017.

Como foi verificado na tabela, todos os alunos afirmaram que os softwares são um recurso facilitador para a aprendizagem de quádricas e que contribuem efetivamente para a aprendizagem desse conteúdo matemático. Indicaram ainda que os softwares podem contribuir para a aprendizagem de outros conteúdos matemáticos e que oferecem benefícios se comparados ao trabalho no papel e no quadro. E por último, afirmaram que o suporte dos softwares educacionais pode contribuir e facilitar a aprendizagem em algumas disciplinas do curso de Licenciatura em Matemática. 
O gráfico a seguir, indica a opinião dos alunos acerca do estudo das superfícies quádricas a partir da utilização dos softwares Geogebra, Winplot e K3DSurf.

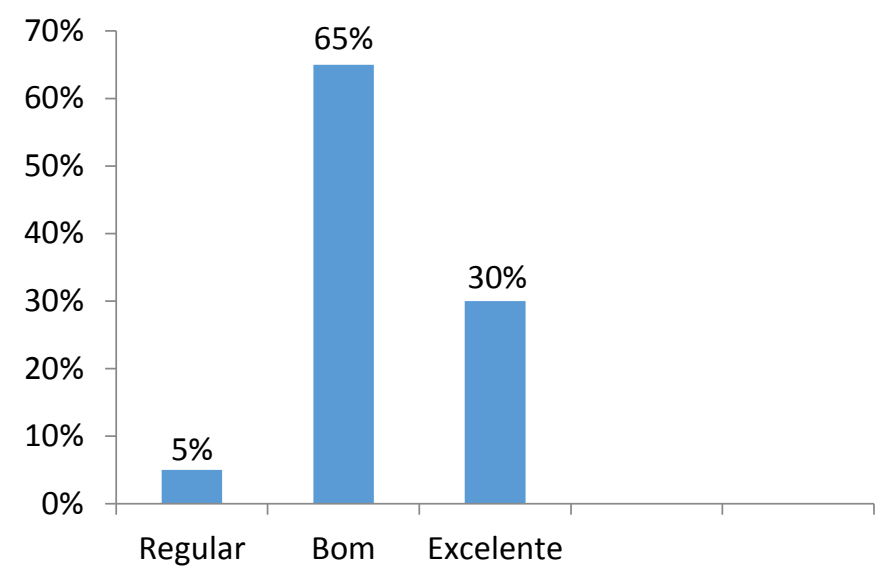

Figura 8 - Gráfico do estudo das superfícies quádricas a partir da utilização dos softwares Geogebra, Winplot e K3DSurf.

De toda amostra da pesquisa, apenas $5 \%$ dos alunos indicou que o estudo proposto foi regular; $65 \%$ afirmaram ter sido bom e $30 \%$ afirmaram que o estudo realizado foi excelente, o que permite a constatação de que quase todos os alunos da amostra, com exceção de um, caracterizaram positivamente o estudo desenvolvido sobre as quádricas.

Assim sendo, constatou-se que a oficina pedagógica desenvolvida com os alunos da disciplina de Cálculo Diferencial e Integral III, trouxe resultados relevantes para o estudo das superfícies quádricas, tendo em vista que todos os alunos participaram ativamente do estudo proposto e demonstraram grande entusiasmo e desenvolvimento diante das atividades propostas.

\section{Considerações finais}

Os estudantes da disciplina de Cálculo diferencial e Integral III apresentaram uma grande aceitação diante do estudo desenvolvido. De modo geral, os softwares educacionais facilitaram a compreensão dos conceitos estudados e a visualização dos gráficos e características das superfícies estudadas, despertando nos discentes a curiosidade de interagir com as suas ferramentas e de aprender a construir objetos e soluções. 
Vale ressaltar, que o professor deve ter a percepção de que nem sempre a utilização de recursos tecnológicos irá contribuir efetivamente com o ensino e aprendizagem de conceitos de Matemática. Acredita-se que os benefícios e vantagens desses tipos de instrumentos didáticos, decorrerão da forma que como serão utilizados, ou seja, para que esses recursos sejam inseridos em sala de aula e surtam efeitos positivos, é necessário que haja um estudo intenso e o planejamento do material que será proposto.

\section{Referências}

BRASIL. Ministério da Educação. Parâmetros Curriculares Nacionais: Matemática /Secretaria de Educação Fundamental. Brasília: MEC/SEF, 1998.

GHIGGI, I. M. F.; SCHMIDT, C. E.; KOCH, B. C. Estudo das cônicas em um ambiente de Geometria Dinâmica. 2013. IV Congresso Internacional de Ensino da Matemática. ULBRA - Canoas, RS. Disponível em: <www.conferencias.ulbra.br/index.php/ciem/vi/paper/view/1150/400>. Acesso em: 02 fev. 2017.

GIL, A. C. Métodos e técnicas de pesquisa social. 6. ed. São Paulo: Atlas, 2008.

GRAVINA, M. A.; SANTAROSA, L. M. A aprendizagem da Matemática em Ambientes Informatizados. Acta do IV Congresso Ibero-Americano de Informática na Educação, Brasília, 1998. Campinas, 2003.

PRODANOV, C. C.; FREITAS, E. C. Metodologia do trabalho científico: métodos e técnicas da pesquisa e do trabalho acadêmico. 2. ed. Novo Hamburgo: Feevale, 2013.

VALENTE, J. A.; ALMEIDA, F. J. Visão Analítica da Informática na Educação no Brasil: a questão da formação do professor. 1997. Revista Brasileira de Informática Educativa [online], Florianópolis, n. 1, p. 1-28. Disponível em: <http://www.scielo.br/scielo.php?script=sci_nlinks\&ref=000160\&pid=S0101$7330201200040000600022 \& \operatorname{lng}=e n>$. Acesso em: 18 jan. 2017.

VALENTIM, E. S. O software Winplot e a prática pedagógica do professor de matemática. 2014. Monografia (Especialização em fundamentos da educação: práticas pedagógicas interdisciplinares). Universidade Estadual da Paraíba. Disponível em: $<$ http://dspace.bc.uepb.edu.br/jspui/bitstream/123456789/4460/1/PDF\%20\%20\%20Eriv an\%20Sousa\%20valentim.pdf>. Acesso em: 20 jan. de 2017. 